\title{
DIFERENCIA ENTRE MÉDICOS Y ODONTÓLOGOS RESPECTO A LA DONACIÓN DEL PROPIO CUERPO PARA DOCENCIA E INVESTIGACIÓN EN CÓRDOBA, ARGENTINA
}

\author{
Susana N. Biasutto ${ }^{1}$, Lucía M. Navarro ${ }^{1}$, Florencia Turri ${ }^{1}$, Nicolás Longoni ${ }^{1}$, \\ Atilio J. Bertocchi Valle ${ }^{2}$, Sofía G. Siemsen ${ }^{1}$, Federico De Oro', Isaías E. Molina \\ Vargas $^{2}$, Oscar P. David ${ }^{3}$, Marcos A. Spinelli ${ }^{1}$, Daniel Urrutia ${ }^{3}$
${ }^{1}$ Cátedra de Anatomía Normal, Facultad de Ciencias Médicas, Universidad Nacional de Córdoba, Córdoba, Argentina
${ }^{2}$ Facultad de Ciencias Médicas, Universidad Nacional de Córdoba, Córdoba, Argentina
${ }^{3}$ Cátedra de Anatomía, Facultad de Odontología, Universidad Nacional de Córdoba, \\ Córdoba, Argentina
}

\section{RESUMEN}

En Argentina, la mayoría de las universidades tienen deficiencia de material cadavérico. Medicina y Odontología son las carreras que utilizan cuerpos en forma regular para la docencia-aprendizaje de la Anatomía. Este hecho ha motivado una consideración particular dentro de nuestro estudio para establecer la actitud de diferentes grupos de población respecto a la donación de cuerpos. Nuestro objetivo es evidenciar las diferencias que observamos entre los profesionales egresados de las dos carreras como participantes fundamentales para implementar un programa de donación. Estudio transversal cualitativo y relacional, basado en encuestas anónimas con respuestas de múltiples opciones y semi-estructuradas, receptadas a azar a médicos y odontólogos. El cuestionario incluyó datos demográficos y otros vinculados a la importancia de los cuerpos para docencia e investigación y la voluntad de donar. Hubo diferencias en la voluntad de donar entre los encuestados de ambas profesiones. No se evidenciaron diferencias entre los dos grupos por sexo, origen geográfico, religión o práctica quirúrgica en su profesión. Si se evidenciaron diferencias significativas dependiendo de la especialidad, la importancia que se le asigna al cadáver para la docencia-aprendizaje de la Anatomía, la actitud hacia la donación de órganos para trasplante y el conocimiento previo sobre la posibilidad de donar el cuerpo. Los médicos muestran mayor tendencia a donar independientemente de la especialidad, porque consideran que los cuerpos son fundamentales para la enseñanza-aprendizaje de la Anatomía y tienen mayor conocimiento sobre donación; lo que influye tanto en la donación de órganos como del cuerpo completo.

Palabras clave: Donación de cuerpos, disección, enseñanza-aprendizaje, voluntad de donar

\begin{abstract}
In Argentina, most of the universities have deficiency of corpses. Medicine and Dentistry regularly use corpses for Anatomy teaching-learning. This fact motivated a particular consideration in our study to determine the attitude of different groups in relation to body donation. Our objective was to show the observed differences between professionals graduated of these both careers as important participants to develop a donation program. This is a qualitative transversal and relational study based on an anonymous survey with multiple choice and semistructured responses. The survey was addressed to physicians and dentists selected by hazard. The questionnaire included demographic data and others associated to the importance of corpses for teachinglearning and the attitude to donation. There were differences among the surveyed professionals in their will to donate. There were no differences by sex, geographic origin, religion or surgical practice. We observed statistical significant differences associated to the speciality, importance assigned to the corpses for Anatomy teaching-learning, the attitude to organs donation for transplantation and knoledge on donation, influencing as well organ and whole body donation.
\end{abstract}

Key words: Body donation, dissection, teachinglearning, will to donate.

* Correspondencia a: Prof. Dr. Susana N. Biasutto. subiasutto@gmail.com

Recibido: 13 de Febrero, 2020. Revisado: 02 de Marzo, 2020. Aceptado: 05 de Marzo, 2020. 


\section{INTRODUCCIÓN}

En Argentina, la mayoría de las universidades presentan una importante deficiencia de material cadavérico. En algunos casos, son cuerpos no reclamados y, en otros, son donaciones libres, voluntarias y altruistas. La Universidad Nacional de Córdoba obtiene la mayoría de los cuerpos por donación de la persona en vida o de sus familiares luego del fallecimiento, pero la cantidad total es insuficiente para la docencia y, consecuentemente, restringe la investigación y docencia de posgrado. Las posibilidades de entrenamiento en prácticas invasivas, formación de posgrado (especialmente quirúrgica) y la investigación se ven seriamente comprometidas por la necesidad de destinar los escasos recursos a la formación de grado.

Medicina y Odontología son las carreras que utilizan material cadavérico en forma regular para la docencia-aprendizaje de la Anatomía y, por ende, solo en sus Cátedras de Anatomía se diseca. Este hecho ha motivado una consideración particular dentro de nuestro estudio para establecer la actitud de diferentes grupos de población respecto a la donación de cuerpos (Biasutto et al., 2018; 2019a; 2019b; 2019c) tendiente al desarrollo de un programa que permita incrementar las donaciones y establecer criterios claros para el manejo de los cuerpos (traslado, registro, almacenamiento, conductas éticas exigidas para su manipulación y utilización, destino final y reconocimiento a los donantes).

En un artículo previo (Biasutto et al., 2019c) hemos publicado la opinión de profesionales médicos (M) y odontólogos (O), que constituyeron uno de los grupos de estudio de nuestro proyecto. En este caso, nuestro objetivo es evidenciar las diferencias que observamos entre los profesionales egresados de las dos carreras (Odontología en Argentina es una carrera y Facultad diferente a Medicina).

\section{MATERIAL Y METODO}

Estudio transversal cualitativo y relacional, basado en encuestas anónimas con respuestas de múltiples opciones y semi-estructuradas, receptadas al azar a médicos y odontólogos. El principal criterio de inclusión fue vivir en la ciudad de Córdoba o, al menos, desarrollar alguna actividad profesional en forma regulare dentro del ámbito de la ciudad. Este requisito se estableció para que correspondieran el área de trabajo con el ámbito de incumbencia que establecería el programa de donación. No hubo criterios de exclusión relacionados a la universidad, lugar o tiempo de graduación, a la especialidad o actividad laboral; excepto los profesores de Anatomía que no fueron incluidos en este grupo. Los profesionales fueron encuestados principal, pero no exclusivamente, en instituciones sanitarias públicas y privadas.

El cuestionario incluyó datos demográficos y otros vinculados a la importancia de los cuerpos para docencia e investigación y la voluntad de donar. Se preguntó edad, sexo, nacionalidad, provincia de origen, religión, profesión, especialidad, si tiene práctica quirúrgica, importancia asignada a los cuerpos para la enseñanzaaprendizaje de la Anatomía, voluntad de donar órganos para trasplante, conocimiento sobre la posibilidad de donar el cuerpo completo, interés en obtener mayor información y voluntad de donar su propio cuerpo.

El análisis estadístico se basó en la distribución porcentual, la determinación de la significación de las diferencias de las respuestas con opciones múltiples; y para las respuestas semiestructuradas se agregó el análisis cualitativo. Las encuestas se analizaron mediante pruebas de $\mathrm{CHI}$ cuadrado, considerándose como significativas la $p<0.05$. Se utilizó el software estadístico Infostat.

Este estudio se realizó con la aprobación y subsidio de la Secretaría de Ciencia y Tecnología de la Universidad Nacional de Córdoba (SECYT-UNC). Proyecto Formar 201819 - 33820180100313CB01

\section{RESULTADOS}

Se encuestaron 429 médicos y 99 odontólogos. La edad de los médicos osciló entre 23 y 81 , con una media de $41,70 \pm 11,62$. La edad de los odontólogos varió entre 23 y 71 , con una media de 40,66 $\pm 10,16(p=<0,0001)$.

De los médicos encuestados $202(47,09 \%)$ eran mujeres y $227(52,91 \%)$ varones, y entre los odontólogos, $53(53,54 \%)$ mujeres y 46 varones $(46,46 \%)$. $(p=0,2471)$.

De los $12(2,80 \%)$ profesionales extranjeros encuestados, todos eran médicos. Provenían 3 de Bolivia, 2 de Perú, 1 de Chile, 1 de Colombia, 1 de Estados Unidos, 1 de México, 1 de Paraguay, 1 del Reino Unido y uno sin especificar. A pesar de no haber odontólogos extranjeros en el grupo y debido a la cantidad tan pequeña, la diferencia comparativa entre las profesiones no es significativa $(p=0,0923)$.

Doscientos noventa y uno $(69,78 \%)$ de los médicos y 72 (72,73\%) de los odontólogos argentinos, eran originarios de Córdoba $(p=0,3435)$. 
En relación a la práctica religiosa, el 284 $(66,20 \%)$ de los médicos y $77(77,78 \%)$ de los odontólogos manifestaron ser católicos; 123 $(28,60 \%)$ médicos y $13(13,13 \%)$ odontólogos refieren no profesar ningún credo; los cristianos no católicos fueron $11(2,56 \%)$ entre los médicos y $5(5,05 \%)$ entre los odontólogos; había 4 $(0,93 \%)$ y $3(3,03 \%)$ judíos entre los médicos y odontólogos respectivamente; en ningún grupo hubo musulmanes, y $7(1,64 \%)$ médicos y 1 $(1,01 \%)$ odontólogo indicaron profesar otra religión. $(p=0,0086)$.

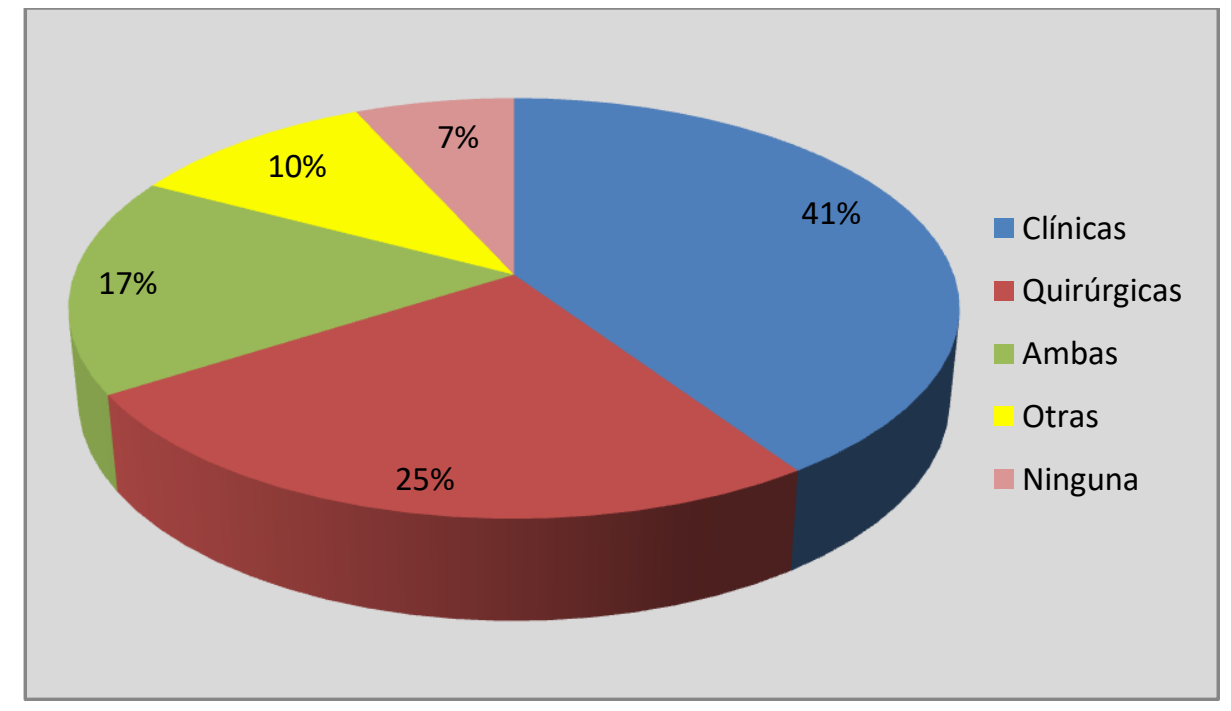

Gráfico 1: Resultados distribuidos según los grupos de especialidades médicas.

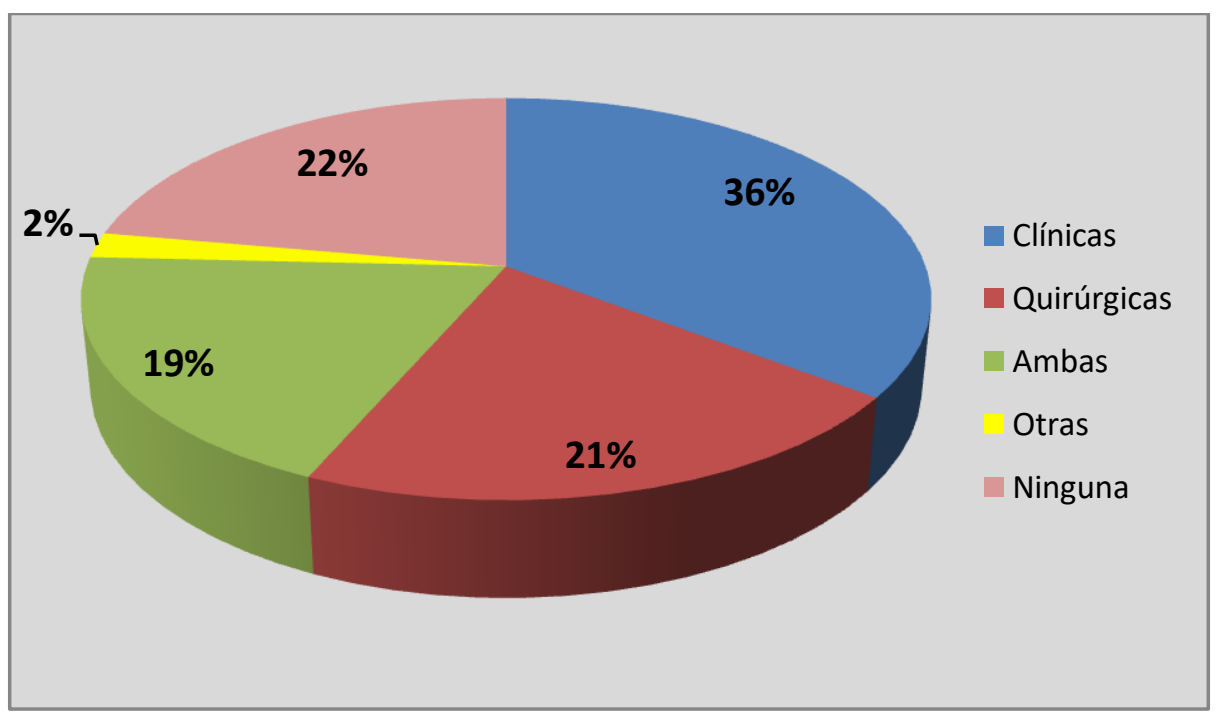

Gráfico 2: Resultados distribuidos según los grupos de especialidades odontológicas

Entre los encuestados se identificaron 60 especialidades médicas diferentes y 15 especialidades odontológicas. Para su mejor evaluación y análisis se agruparon en: 1) Clínicas, 2) Quirúrgicas, 3) Ambas (especialidades que tienen tratamientos clínicos y quirúrgicos dentro de la patología propia. Ej.: Oftalmología, Otorrinolaringología, etc.), 4) Otras (aquellas especialidades no clínicas ni quirúrgicas, como Auditoría, Medicina Legal, Medicina Laboral, etc.) y 5) Ninguna (profesionales que refieren no ser especialistas). La distribución de estos grupos puede verse en los gráficos 1 y 2 , y la diferencia resulta altamente significativa entre los profesionales de ambas carreras $(p=<0,0001)$. 
Cuarenta y cinco por ciento (194) de los médicos y $79 \%$ de los odontólogos refirieron tener práctica quirúrgica, lo que determina una significativa diferencia a favor de los últimos $(p=<0,0001)$.

En relación a la importancia que estos profesionales asignan al material cadavérico para la enseñanza-aprendizaje de la Anatomía, el $93,47 \%$ de los médicos consideraron que "mucho", al igual que el $91,92 \%$ de los odontólogos. El 0,47\% de los médicos y el 2,02\% de los odontólogos eligieron "nada"; y los restantes le asignaron "poca" importancia $(6,06 \%$ para ambos grupos de profesionales). $(p=0,2746)$

Médicos y odontólogos manifiestan su voluntad de donar órganos en un $94,64 \%$ y $88,89 \%$ respectivamente. $(p=0,0045)$

El $68,30 \%$ de los médicos y el $57,58 \%$ de los odontólogos refieren conocer acerca de la posibilidad de donar el propio cuerpo para docencia e investigación $(p=0,0419)$, mientras que el $67,60 \%$ y $61,62 \%$, respectivamente, estarían interesados en disponer de mayor información ( $p=0,2557)$.

Doscientos dieciséis $(50,35 \%)$ médicos y 39 $(39,39 \%)$ odontólogos donarían sus cuerpos $(\mathrm{p}=0,0298)$.

Respecto a la donación, no se observaron diferencias por sexos $(p=0,2797)$. Si bien en ambas profesiones los varones mostraron porcentajes ligeramente mayores que las mujeres en cuanto a su disposición a donar el cuerpo, estos valores no alcanzaron a ser estadísticamente significativos.

El $50 \%$ de los extranjeros expresó una actitud positiva hacia la donación, sin embargo, no se pudo realizar análisis comparativo porque todos los encuestados eran médicos.

Los médicos no originarios de Córdoba expresaron mayor voluntad de donar $(55 \%)$ que los cordobeses (48\%), mientras que los odontólogos cordobeses manifestaron mejor actitud hacia la donación (43\%) que los nocordobeses $(30 \%)$. Sin embargo, estas diferencias no fueron estadísticamente significativas sea dentro de cada grupo $(p=0,2771$ y $p=0,1700$ respectivamente) o entre grupos ( $p=0,1031)$.

La voluntad de donar fue más frecuente entre quienes no profesan ninguna religión $(60 \%$ en los médicos y $54 \%$ en los odontólogos con actitud positiva a la donación), pero estas cifras no alcanzaron diferencia estadísticamente significativa por religiones $(p=0,0555)$, ni entre los dos grupos de profesionales $(p=0,1513)$, a pesar que su composición tiene un espectro distinto como se describió más arriba.

La intención de donar en relación a las especialidades en las dos profesiones fue diferente $(p=<0,0001)$. Los médicos se manifestaron en porcentajes similares en los grupos por especialidad, mientras que los odontólogos clínicos tuvieron mayor disposición hacia la donación.

Los médicos sin práctica quirúrgica y los odontólogos con práctica quirúrgica fueron los que se expresaron más favorablemente en relación a la donación de sus propios cuerpos. Estos resultados, sin embargo, no tuvieron diferencias significativas $(p=0,2818)$.

Cuando los profesionales respondieron que los cuerpos son "muy" importantes para la docenciainvestigación el $53 \%$ de los $\mathrm{M}$ y el $40 \%$ de los $\mathrm{O}$ estaba dispuesto a donarse. Quienes respondieron que los cadáveres eran "poco" importantes invirtieron su voluntad de donar el propio cuerpo, $19 \%$ para $\mathrm{M}$ y $50 \%$ para $\mathrm{O}$, aunque las cifras absolutas fueron bajas. En cambio, los pocos que respondieron que no son importantes $(3 \mathrm{M} \mathrm{y}$ 2 O), en un $100 \%$ respondieron que no donarían. Todo ello determinó que la diferencia fuera estadísticamente significativa $(\mathrm{p}=0,0030)$.

El $53 \%$ de los médicos y el $43 \%$ de los odontólogos que donarían sus órganos para trasplante, también donarían su cuerpo para docencia e investigación $(p=0,0003)$.

Los médicos que tenían conocimiento sobre la donación de cuerpos manifestaron mayor voluntad de donarlos $(55 \%$ - $p=0,0071)$ que los odontólogos en quienes no influyó el conocimiento previo $(p=0,9161)$. Esto determinó que la diferencia entre ambos grupos de profesionales fuera estadísticamente significativa $(p=0,0151)$.

\section{DISCUSIÓN}

Analizando nuestros resultados surge que no hubo diferencias en la voluntad de donar entre los encuestados de ambas profesiones a pesar de los porcentajes distintos. Tampoco se evidenciaron diferencias entre los dos grupos por sexo, origen geográfico, religión o práctica quirúrgica en su profesión. Si se evidenciaron diferencias significativas dependiendo de la especialidad, la importancia que se le asigna al cadáver para la docencia-aprendizaje de la Anatomía, la actitud hacia la donación de órganos para trasplante y el conocimiento previo sobre la posibilidad de donar el cuerpo. Los médicos muestran mayor tendencia a donar independientemente de la especialidad, porque consideran que los cuerpos son fundamentales para la enseñanza-aprendizaje de la Anatomía y tienen mayor conocimiento sobre donación; lo que influye tanto en la donación de órganos como del cuerpo completo. 
La mayor parte de las publicaciones sobre el tema se basan en encuestas realizadas a docentes y profesores de Anatomía (ArraezAybar et al., 2010; Anyanwu and Obikili, 2012; Emue et al., 2012; Quiroga-Garza et al., 2017), quienes fueron específicamente excluidos en nuestro estudio.

Saha et al. (2015) public un grupo de 100 médicos, con igual cantidad de mujeres $y$ varones, que muestra una diferencia significativa con los $M$ de nuestro trabajo tanto para trasplante de órganos $(86 \%-\mathrm{p}=0,0023)$ como para donación del cuerpo complete (26\% $p=<0,0001)$.

Otro trabajo basado en la actitud de los médicos fue publicado por Ballala et al. (2011) con encuestas a 89 personas (57\% varones y $43 \%$ mujeres), orientado principalmente a aspectos formales de la donación, pero en el que podríamos señalar algunos aspectos interesantes. En este estudio solo el $8 \%$ no conocía acerca de la posibilidad de donar, a diferencia del $32 \%$ en nuestro trabajo $(p=<0,0001)$, pero solo el $25 \%$ donaría su propio cuerpo $(p=<0,0001)$ siendo que el $89 \%$ consideró que el public general debería donar para contribuir con el progreso de la Medicina.

Green et al. (2014) estudió un grupo de médicos del Hospital Universitario de St Vincent en Irlanda, para quienes el $83 \%$ considera fundamental la disección para su educación y, por ende, comparable al 93\% de nuestro estudio que consideró muy importante el cadáver para la enseñanza-aprendizaje de la Anatomía $(p=0,0002)$. La actitud hacia la donación de órganos para trasplante $(p=0,0687)$ y la donación del propio cuerpo $(p=0,4882)$ fue similar en ambos estudios.

No hemos hallado publicaciones que involucren específicamente a los odontólogos en la literatura.

De nuestro trabajo se puede concluir que la actitud de los profesionales médicos y odontólogos es positiva para contribuir con el desarrollo de un programa de donación y, comparativamente con la literatura, mejor que en otras publicaciones. De las diferencias observadas en nuestros resultados podríamos remarcar la importancia de proveer mayor información como parte de las respectivas carreras, en especial en Odontología.

\section{Conflicto de interés \\ Ninguno}

\section{Financiamiento}

Este trabajo fue aprobado y subsidiado por el Programa 2018-19 de la SECYT-UNC 33820180100313 CB01.

\section{Aprobación Ética \\ No necesaria}

\section{Consentimiento informato}

Los encuestados fueron informados acerca de los objetivos del proyecto y, en todos los casos, su participación fue estrictamente voluntaria.

\section{Contribuciones}

SNB: Diseño del proyecto, directora, bibliografía, prepararción y coordinación de las encuestas, registro y procesamiento de datos, análisis estadístico y redacción del manuscrito. LMN: Preparación y recepción de encuestas. Diseño gráfico FT: Preparación y recepción de encuestas. Diseño gráfico. NL: Preparación y recepción de encuestas. Diseño gráfico. AJBV: Preparación y recepción de encuestas. Diseño gráfico. SGS: Preparación y recepción de encuestas. Diseño gráfico. FDO: Preparación y recepción de encuestas. Diseño gráfico. IEMV: Preparación y recepción de encuestas. Diseño gráfico. OPD: Diseño del proyecto, vice-director del proyecto, bibliografía, preparación de la encuesta, y recepción de datos. MAS: Diseño del proyecto, preparación y recepción de encuestas. DU: Diseño y preparación de la encuesta.

\section{AGRADECIMIENTOS}

Agradecemos a todos aquellos que voluntariamente donan su cuerpo para la docencia e investigación universitaria.

\section{BIBLIOGRAFÍA}

Anyanwu EG, Obikili EN. 2012. Dissecting the Dissectors: Knowledge, Attitude, and Practice of Body Bequests by Nigerian Anatomists. Anatomical Science Education 5: 347-53.

Arraez-Aybar LA, Sanchez-Montesinos I, Mirapeix RM, Mompeo-Corredera B, SanudoTejero JR, 2010. Relevance of human anatomy in daily clinical practice. Ann. Anat. 192: 34148.

Ballala K, Shetty A, Malpe SB. 2011. Knowledge, attitude, and practices regarding whole body donation among medical professionals in a hospital in India. Anat Sci Educ 4: 142-50.

Biasutto SN, Garay MB, Rives MV, Uanini $F$, Albrecht A, Ortiz LB, Gerbaldo MV. 2018. La percepción de los estudiantes de primer año de Medicina en la sala de disección y su incidencia sobre la procuración de cuerpos. Rev Arg de Anat Clin 10: 44-51 
Biasutto SN, Molina Vargas IE, Weigandt DM, Mora MV, Vargas RAA, Bertocchi Valle AJ, Uanini MF, David OP, Urrutia $D$, Spinelli $M A$, Olivera MNJ, Gimenez M, Nefa Salera M, Decouvette A, Ferrero LJ. 2019a. Reactions of first year medical students in the dissection room, with prosected corpses, and the incidence on own body donation. Rev Arg de Anat Clin 11: 18-29

Biasutto SN, Spinelli MA, Weigandt DM, Mora MV, Bertocchi Valle AJ, Vargas RAA, Molina Vargas IE, David OP, Urrutia D. 2019b. Attitude of medical students to body donation in the National University of Cordoba - Argentina. Rev Arg de Anat Clin 11: 70-83.

Biasutto SN, David OP, Spinelli MA, Urrutia D, Bertocchi Valle AJ, Weigandt DM, Molina Vargas IE, Vargas RAA, Navarro LM, Siemsen $S G$, Turri F, Longoni N, De Oro F, Prost SA. 2019c. Research on body donation willingness in Cordoba-Argentina: medical and dentist doctors' attitude. Rev Arg de Anat Clin 11: 10714.
Emue BEB, Jacks TW, Danladi SA, Nwegbu MM, Abue $A D$, Onwih EE. 2012. Problems and prospects of acquistion of human cadaver for medical education in Nigeria. J Pak Med Assoc 62: 1134-36.

Green C, Bowden D, Molony D, Burke N, Felle P, Dudeney S. 2014. Attitudes of the medical profession to whole body and organ donation. The Surgeon 12: 73-77

Quiroga-Garza A, Reyes-Hernandez CG, ZarateGarza PP, Esparza-Hernandez CN, Gutierrezde la $O \mathrm{~J}$, de la Fuente-Villarreal $D$, ElizondoOmana RE, Guzman-Lopez S. 2017. Willingness toward organ and body donation among anatomy professors and students in Mexico. Anat Sci Educ 10: 589-97.

Saha A, Sarkar A, Mandal S. 2015. Body donation after death: The mental setup of educated people. Journal of Clinical and Diagnostic Research 9: 5-9.

Sugand K, Abrahams P, Khurana A. 2010. The anatomy of anatomy: a review for its modernization. Anat Sci Educ 3: 83-93. 